\title{
Review \\ Current applications and future direction of MR mammography
}

\author{
PJ Kneeshaw', LW Turnbull ${ }^{2}$ and PJ Drew*, \\ 'Academic Surgical Unit, Castle Hill Hospital, Castle Road, Cottingham HU I 6 5JQ, UK; ${ }^{2}$ The Centre for Magnetic Resonance Investigations, Hull Royal \\ Infirmary, Hull, UK
}

\begin{abstract}
Compared with triple assessment for symptomatic and occult breast disease, magnetic resonance mammography (MRM) offers higher sensitivity for the detection of multifocal cancer, which is important in selecting patients appropriately for breast-conserving surgery. It is an ideal tool for the screening of patients with a high risk of breast cancer or where there is axillary disease or nipple discharge and conventional imaging has not revealed the primary focus. Techniques are now available to biopsy lesions only apparent on MRM. MRM can differentiate scar tissue from tumour; therefore, it is useful in patients in which there is possible recurrent disease. Clinical and X-ray mammographic assessment of response to neoadjuvant chemotherapy may be unreliable because of replacement of the tumour with scar tissue. MRM can identify responders and nonresponders with more accuracy. It is the modality of choice for the assessment of breast implants for rupture with accuracy higher than X-ray mammography and ultrasound. Advances in both spatial and temporal resolutions, the imaging sequences employed, pharmacokinetic modelling of contrast uptake, the use of dedicated and now phased-array breast coils, and gadolinium-based contrast agents have all played their part in the advancement of this imaging technique. Despite the limitations of patient compliance, scan-time and cost, this review describes how MRM has become a valuable tool in breast disease, especially in cases of diagnostic uncertainty. However, MRM must make the transition from research institutions into routine clinical practice.
\end{abstract}

British Journal of Cancer (2003) 88, 4- 10. doi:10.1038/sj.bjc.66007/3 www.bjcancer.com

(c) 2003 Cancer Research UK

Keywords: magnetic resonance mammography; breast imaging

The use of magnetic resonance imaging (MRI) in the evaluation of breast disease has continued to gain recognition over the last 15 years, although mainly in research institutions. However, despite its apparent advantages, magnetic resonance mammography (MRM) still awaits introduction into routine clinical practice.

MRM relies on the presence of well-established morphological features that help distinguish malignant from benign lesions. In addition, angiogenesis induced by cancers is demonstrated by dynamic contrast-enhanced magnetic resonance imaging (DCEMRI). Areas of increased microvessel density are delineated following intravenous gadolinium - diethylenetriaminepentaacetic acid (Gd-DTPA). The enhancement in malignant tissue is thought to be because of increased permeability, vascularity and increased interstitial space (Vaupel, 1994) (Figure 1)

In dynamic imaging areas of parenchymal deformity in the breast may be re-imaged over time, thus allowing the signalintensity - time curve to be analysed in great detail. Fast scanning techniques now make it possible to image preselected areas of the breast at least every $2 \mathrm{~s}$. Curve analysis can be carried out by a number of techniques, most typically by empirical methods or pharmacokinetic modelling.

Kuhl et al reported a correlation between the shapes of the signal-intensity curves and the likely aetiology. The curves were subdivided into four categories. Types Ia or Ib are typical of benign lesions, and types II and III are consistent with malignant lesions. Using this method, Kuhl reported a sensitivity of $91 \%$, a

*Correspondence: Mr PJ Drew; E-mail: pjdrew@hull.ac.uk specificity of $83 \%$ and an accuracy of $86 \%$ in distinguishing benign from malignant lesions (Kuhl et al, 1999) (Figure 2).

Pharmacokinetic modelling is a mathematical process that uses all the signal intensity data to give numeric values of the permeability and the contrast exchange rate between the plasma and the extravascular and extracellular space of a lesion. These data can be used to differentiate benign from malignant lesions objectively (Buckley et al, 1994) (Figure 3).

\section{SYMPTOMATIC DISEASE}

Currently, triple assessment is the gold standard for symptomatic breast disease. In our centre, we have compared the evaluation of 285 symptomatic patients presenting to the breast clinic by both the triple assessment and MRI. The sensitivity of each modality was as follows: clinical examination $84 \%$, mammography $87.6 \%$, fine-needle aspiration cytology $79.1 \%$, triple assessment $99.2 \%$ and MRI $99.2 \%$. In addition, histologically confirmed multifocal disease was detected by MRI in 40 patients, but in only nine patients $(22.5 \%)$ on mammography. The specificity for the diagnosis of benign disease was as follows: clinical examination $83.1 \%$, ultrasound $88.9 \%$, mammography $86.4 \%$, fine-needle aspiration cytology $97 \%$, triple assessment $59.1 \%$ and MRI $90.9 \%$. We concluded that DCE-MRI of the breast is as sensitive and more specific than the combined traditional triple assessment for the diagnosis of malignant breast lesions (Drew et al, 1999b). Similar findings have been reported in several other studies (Table 1). 

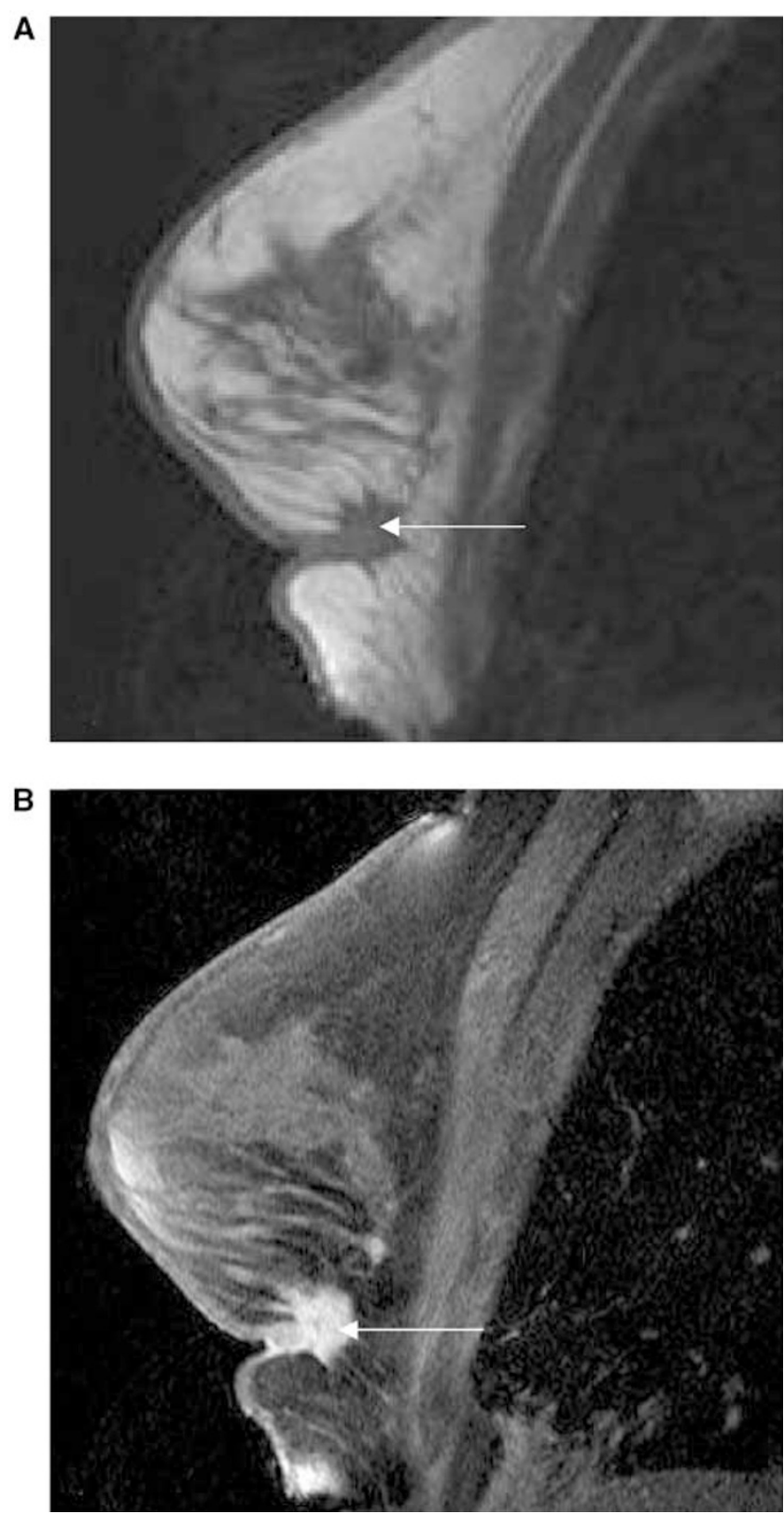

Figure I A TI-weighted image of an invasive breast cancer $(\mathbf{A})$ fatsaturated image after gadolinium contrast (B)

\section{OCCULT DISEASE}

The accurate staging of primary breast cancer is paramount. The surgical plan depends upon the size and position of the mass. The presence of multiple tumours in the same quadrant (multifocal) or in multiple quadrants (multicentric) must be determined. Mammography is still the main imaging modality in breast cancer with sensitivity between 69 and $90 \%$ and the specificity ranging from 10 to $40 \%$ (Rankin, 2000). Tumours may be missed because of observer error, dense breast tissue or even poor technique. Using dynamic MRI of the breast, sensitivities are in excess of $95 \%$, with specificities over $80 \%$ (Turnbull, 2000). In a recent study by Fischer et al, $16 \%$ of candidates for breast-conserving surgery had clinically relevant findings at MR examination (Fischer et al, 1999). In a similar but smaller study, Tan et al analysed the impact of MRI on the surgical management of 83 patients being considered

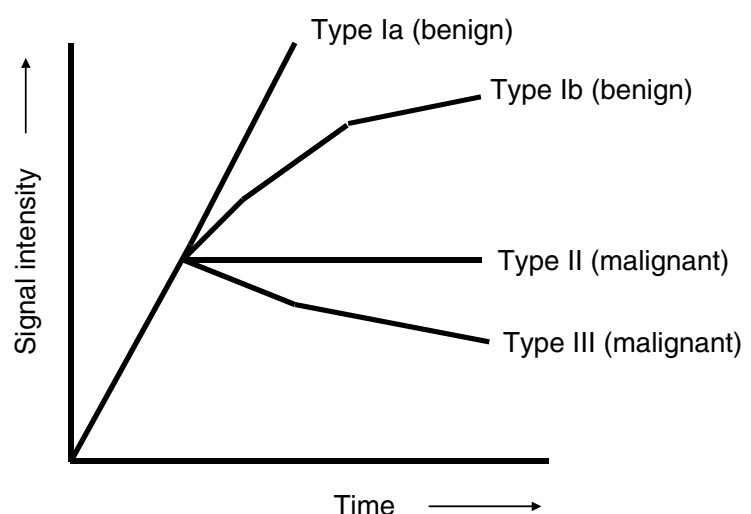

Figure 2 Shapes of signal-intensity curves and likely histology (Kuhl et al, 1999).

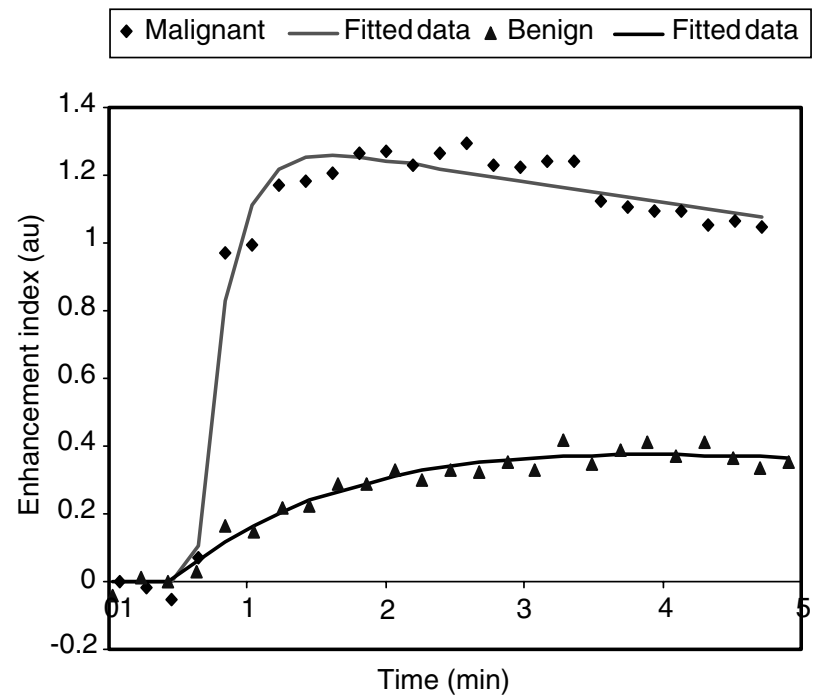

Figure 3 Typical malignant and benign lesion signal-intensity curves (data points) and two-compartment pharmacokinetic modelling (lines).

for breast-conserving surgery. MRI altered the management in $18 \%$ of the patients (Tan et al, 1999).

We evaluated the accuracy of triple assessment compared with MRI for the detection of multifocal disease prior to breast surgery. The resultant sensitivity, specificity, positive and negative predictive values were $18,100,100$ and $76 \%$ for triple assessment and $100,86,73$ and $100 \%$ for DCE-MRI. The study also identified a subgroup of breast cancer patients with multifocal/multicentric disease not evident on standard triple assessment (Drew et al, 1999a).

\section{COMICE TRIAL}

The clinical relevance of preoperative detection of small additional carcinomas by MRI, especially small foci of DCIS has yet to be answered. Should these cancers be excised or would adjuvant radiotherapy treat them satisfactorily? The UK-based comparative effectiveness of MR imaging in breast cancer (COMICE) trial has just commenced. This is a randomised control trial recruiting patients planned for wide local excision for primary breast cancer into two study arms. One group of patients will undergo standard imaging and the other group will have additional preoperative MRI staging. This trial will also clarify whether patients scheduled for conservative breast surgery are being adequately staged as the 
Table I Selected published results of breast MRI

\begin{tabular}{|c|c|c|c|c|c|}
\hline Author & Year & Number of lesions & Number of cancers & Sensitivity & Specificity \\
\hline Heywang (Heywang-Kobrunner et al, 1989) & 1989 & 150 & 71 & 0.98 & 0.65 \\
\hline Kaiser (Buckley et al, 1997) & 1989 & 191 & 58 & 1.0 & 0.97 \\
\hline Orel (Orel et al, 1995) & 1995 & 166 & 64 & 0.92 & 0.88 \\
\hline Bone (Brown et al, 2000) & 1997 & 238 & 145 & 0.92 & 0.72 \\
\hline Drew (Drew et al, 1998) & 1998 & 105 & 9 & 1.0 & 0.93 \\
\hline Lui (Liu et al, 1998) & 1998 & 120 & 70 & 0.93 & 0.74 \\
\hline
\end{tabular}

average re-excision rate nationally is currently $14.2 \%$ (BASO Specialist Group meeting, 2000).

Screening X-ray mammography has resulted in the detection of increasing numbers of $\mathrm{T}_{1 \mathrm{~b}}$ breast lesions, tumour diameter $>0.5 \mathrm{~cm}$ and $\leqslant 1 \mathrm{~cm}$ (Beahrs, 1992). There are little data in the literature regarding the accuracy of diagnosis in sub- $\mathrm{T}_{1 \mathrm{~b}}$ breast lesions by any of the routinely used imaging modalities, namely $\mathrm{X}$ ray mammography, ultrasound or MRI. In a recent study of 63 patients, we evaluated the accuracy of diagnosis of clinically occult sub- $1 \mathrm{~cm}$ lesions using DCE-MRI and achieved a sensitivity of $93.2 \%$ and a negative predictive value of $84.6 \%$ in differentiating benign from malignant sub-1 cm lesions (Kneeshaw et al, 2001a). Therefore, DCE-MRI may have a role following failed stereotactic biopsy or ultrasound-guided core biopsy in patients with very small lesions instead of proceeding to open localised surgical biopsy.

\section{MR-GUIDED BREAST BIOPSY AND LOCALISATION}

The high sensitivity of MRI sometimes means that abnormalities in the breast are detected that cannot be imaged using X-ray mammography or ultrasound. Therefore, MR-guided biopsy techniques are currently being developed. Open configuration scanners allow continuous access to the patient but are limited by low field strength which compromises signal-to-noise ratio (Gould et al, 1998). Heywang-Kobrunner et al described a unilateral openbreast coil. This allowed successful hook wire placement in 10 out of 11 patients with an average lesion size of $12 \mathrm{~mm}$ (HeywangKobrunner et al, 1994). Closed magnet systems allow access to the patient outside the bore of the magnet with a localisation device. Early small studies using freehand and localisation devices have been encouraging. Daniel et al managed to obtain diagnostic tissue in all 27 lesions using a freehand method. Liney et al (2000) advocated the use of carbon-fibre-core biopsy needle to reduce artefact. In a larger series of 59 patients with lesions only visible on MRM, Kuhl et al (2001) achieved a diagnostic accuracy of $98 \%$ using MR-guided stereotactic large-core (14 G) needle biopsy.

\section{SCREENING}

Breast MRI, with its high sensitivity, is potentially an ideal tool for the screening of high-risk populations. It is especially useful in younger women because of the lower sensitivity of X-ray mammography in this group. Stoutjesdijk et al published results in 2001 comparing MRI and mammography in women with a hereditary risk of breast cancer. In a retrospective study of 179 women at high risk of breast cancer, MRI demonstrated 13 cancers, seven of which were not demonstrated by mammography. Receiver operator curves areas for MRI and mammography were 0.99 and 0.74 , respectively (Stoutjesdijk et al, 2001). Warner et al (2002) compared MRI, mammography and ultrasound for surveillance of 196 women with proven BRCA1 or BRCA2 mutations or strong a strong family history of breast cancer. All six invasive breast cancers were picked up by MRI with mammography and ultrasound detecting 2 and 3, respectively (Warner et al, 2002).

The UK magnetic resonance imaging for breast screening (MARIBS) trial is a multicentre ongoing trial comparing X-ray mammography and MRI as a method for screening genetically high-risk women. Women aged 35-50 years with BRCA1 or BRCA2 or aged 25-50 years with TP53 are being recruited. Furthermore, this study is examining women's attitudes to MR examination of the breast (Brown et al, 2000).

\section{RESPONSE TO CHEMOTHERAPY}

Patients with locally advanced disease at presentation are more commonly undergoing neoadjuvant chemotherapy followed by surgery. It is the aim of the treatment to shrink the tumour to allow subsequent mastectomy or breast-conserving surgery (Jacquillat et $a l, 1989)$. Evaluation of the response to chemotherapy by conventional imaging methodology is difficult. This is because of changes induced in the breast parenchyma, including the replacement of tumour with diffuse fibrosis. The fibrotic tissue may be confused with residual tumour on palpation. Breast MRI is more effective than X-ray mammography at determining the extent of residual disease following neoadjuvant chemotherapy (Esserman et al, 1999; Drew et al, 2001) (Figure 4).

Tissue permeability may be evaluated using dynamic breast MRI and this can be used to predict patients who will respond to chemotherapy (Hayes et al, 2002). Predicting tumour response may allow drugs to be altered early in treatment.

\section{DCIS}

The advent of breast screening has shown that in situ disease with or without microinvasion represents $20-25 \%$ of all screendetected breast cancer (Roberts et al, 1990). The MR appearance of microcalcification, the cardinal mammographic feature of DCIS is a signal void. However, in a similar manner to invasive disease, DCIS induces angiogenesis and this can be detected in vivo by DCE-MRI (Figure 5). In a study by Gilles et al (1995), dynamic MRI showed contrast enhancement in 34 out of 36 patients with DCIS. Other MR studies have reported variable accuracy for classification of microcalcification. Westerhof et al (1998), investigating mammographically suspicious microcalcifications, reported a sensitivity of $45 \%$, specificity of $72 \%$, positive predictive value of $71 \%$, negative predictive value $46 \%$ and an accuracy of $56 \%$, and in a further study, Gilles et al observed a sensitivity of $95 \%$ and a specificity of $51 \%$. Their specificity was impaired because the presence or absence of contrast uptake in the breast was the only parameter used to decide if the area of microcalcification was associated with malignancy or not (Gilles et al, 1996).

Malich et al looked specifically at 100 mammographically suspicious lesions using different imaging modalities. He showed 

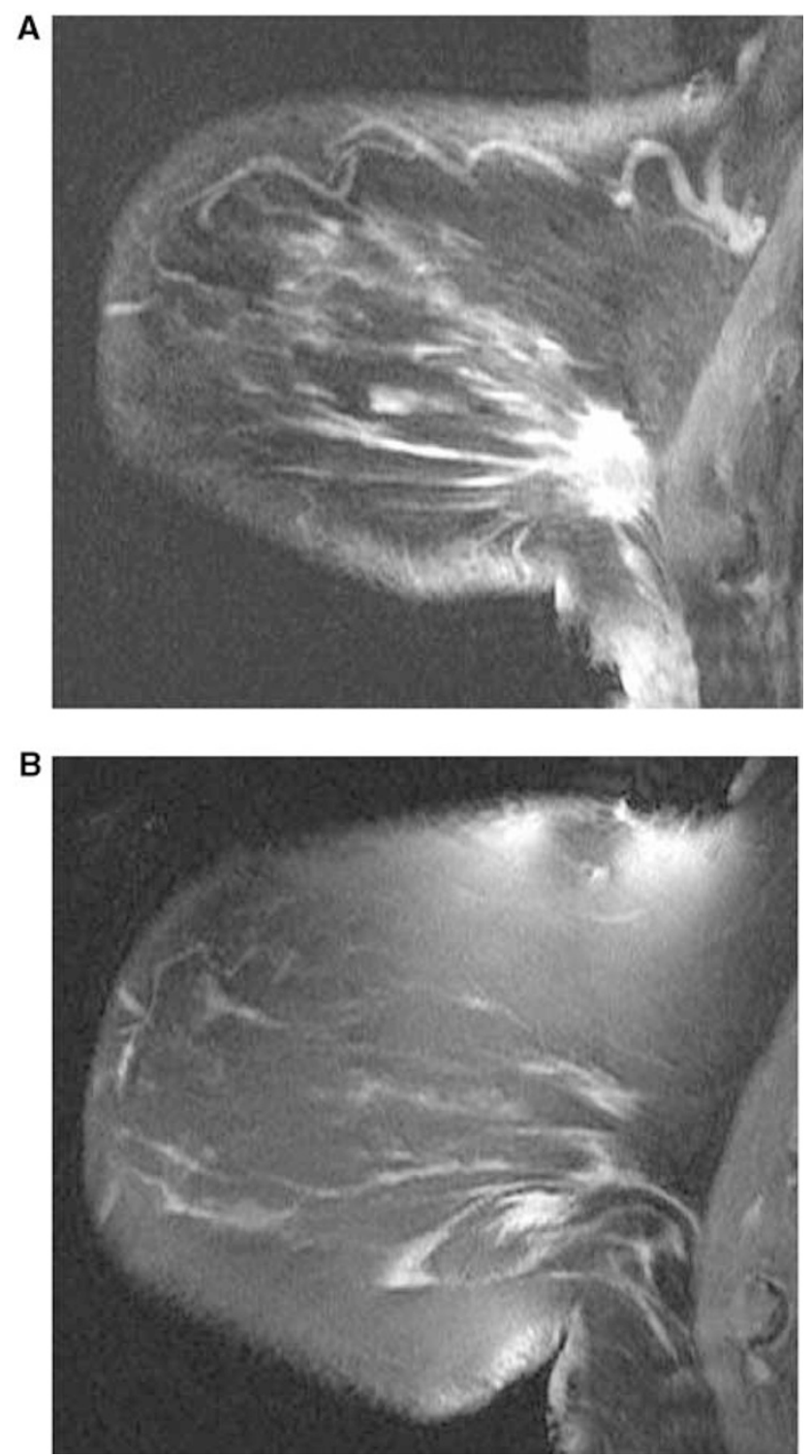

Figure 4 Monitoring the response to neo-adjuvant chemotherapy. Before treatment $(\mathbf{A})$ and response after 3 months $(\mathbf{B})$.

MRI to have a sensitivity of $98 \%$, specificity of $81 \%$, positive predictive value of $88 \%$ and negative predictive value of $97 \%$ for malignant disease, while the corresponding values for DCIS and hyperplasia were 86, 83, 75 and 91\%, respectively (Malich et al, 2001).

We are currently evaluating the use of DCE-MRI in the assessment of screening-detected mammographically indeterminate microcalcifications and initial results for differentiating benign from malignant causes are encouraging. In a cohort of 42 patients, we achieved a sensitivity of $87.5 \%$ and a negative predictive value of $80 \%$ (Kneeshaw et al, 2001b).

\section{RECURRENT DISEASE}

Areas of scar tissue following breast-conserving surgery can simulate recurrences on mammography. MRI performed up to 1year post-surgery shows some enhancement in the scar tissues at dynamic examination making the differentiation of scar from
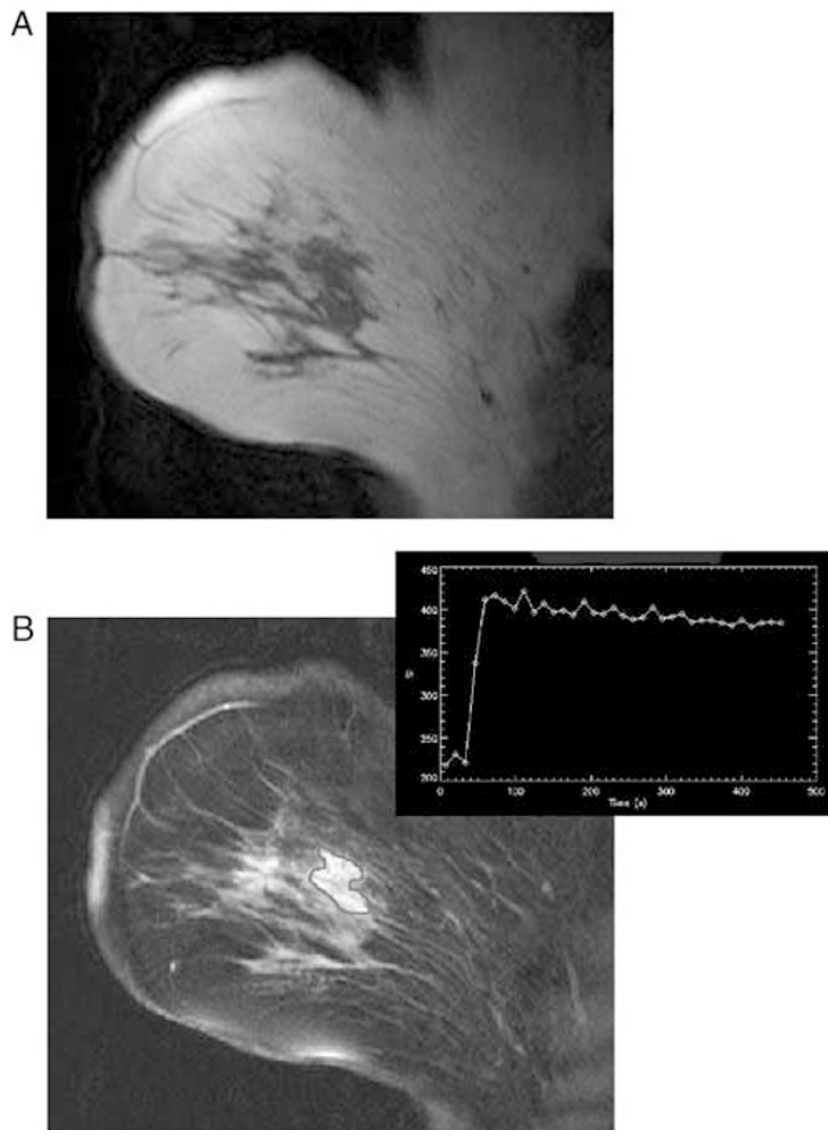

Figure 5 TI-weighted image of DCIS $(\mathbf{A})$ and fat-saturated image after gadolinium contrast (B) with malignant signal-intensity curve of enclosed area.

recurrence unsatisfactory. After 12 months, however, MRI can be used to detect or exclude recurrent tumour with high sensitivity. Kramer et al (1998) reported a sensitivity of $91 \%$ for detection of cancer recurrence with MRI compared to 51, 67 and $83 \%$ for physical examination, X-ray mammography and ultrasound, respectively, in 33 cases of tumour recurrence. We evaluated clinical examination, mammography and MRI in the detection of recurrent tumour following breast-conserving surgery. The sensitivity of clinical examination, mammography, examination combined with mammography, and MRI alone for the detection of recurrent cancer were $89,67,100$ and $100 \%$, respectively, with specificity values of $76,85,67$ and $93 \%$. We concluded that combined clinical examination and mammography were as sensitive as dedicated dynamic MR of the breast for the detection of locoregional recurrence, but that breast MRI was associated with a far greater specificity (Drew et al, 1998).

This year Belli et al evaluated 40 women with clinical or radiological evidence of local recurrence (Belli et al, 2002). MRI identified all the 22 cancers and showed 95\% accuracy, $100 \%$ sensitivity, $88.8 \%$ specificity with $5 \%$ false positives and $100 \%$ negative predictive values.

There are no studies to date evaluating the use of MR for monitoring patients after breast-conserving surgery. However, the Board of the Faculty of Clinical Radiology from The Royal College of Radiologists in their Guidance on Screening and Symptomatic Breast Imaging published 1999 stated that where conventional triple assessment has been unhelpful in the assessment of patients with suspected recurrences, MRI has been shown to be useful. 


\section{AXILLARY DISEASE}

Breast cancer may present as an axillary metastasis with normal triple assessment. MRI has been used in this setting to detect the primary cancer. In one such study Orel et al (2000b) demonstrated the primary cancer in 19 out of 22 patients who presented with an axillary metastasis.

Clinical examination is generally poor in evaluating the axilla for recurrent tumour. Bradley et al evaluated the accuracy of axillary MRI in distinguishing between recurrent tumour and treatmentinduced effects. In their series using MRI, the specificity and sensitivity for the detection of axillary metastasis was 100 and $89 \%$, respectively (Bradley et al, 2000).

\section{NIPPLE DISCHARGE}

Nipple discharge, although usually benign in origin, causes concern because of the possibility of an underlying cancer. Worrying features such as spontaneous, unilateral discharge in which the fluid is bloody, serous or clear warrants further evaluation. In a study by Orel et al (2000a), the use of MRI in the investigation of nipple discharge was evaluated. Mammographic findings proved negative in 22 out of 23 patients while in $73 \%$ of the patients, MR findings correlated with the pathology findings. They concluded that MR imaging could help identify both malignant and benign causes of nipple discharge (Orel et al, 2000a).

\section{BREAST IMPLANTS}

MRI is widely regarded as the investigation of choice for the demonstration of intracapsular and extracapsular breast implant ruptures. The sensitivity of MR for the detection of implant rupture is around $95 \%$ with a specificity of $90 \%$ and is considerably better than mammography or ultrasound (Turnbull, 2000). The intracapsular rupture is recognised by the presence of the linguine sign, which is caused by extensive folding of the collapsed shell of the implant (Gorczyca et al, 1994). Free silicone may be seen in the parenchyma of the breast following an extracapsular tear with silicone-specific MRI sequences (Figure 6).

\section{PRACTICAL LIMITATIONS}

The patient is required to remain still within the scanner for around $20 \mathrm{~min}$ for a standard dynamic MR series. Patients who are

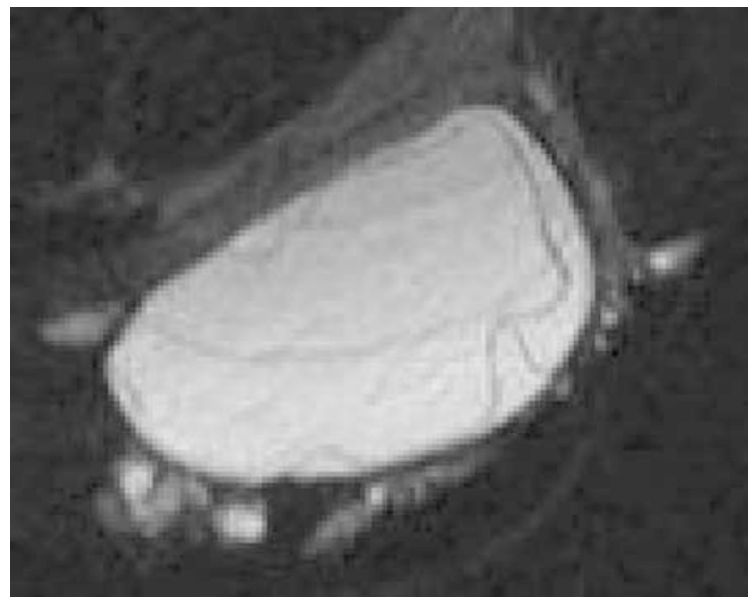

Figure 6 Silicone-specific series showing an extracapsular tear with silicone globules in soft tissues. large or have other medical problems may not tolerate these conditions reducing the quality of the examination. Careful and considerate patient positioning may help to overcome these problems.

Patients with claustrophobia may initially refuse to enter the scanner. Careful explanation about the examination often helps to overcome these problems. Breast scanning is generally performed with the patient prone and the breasts positioned in the cups of the dedicated breast coil. This position is often well tolerated by anxious patients, as they can see out of the magnet, but in severe cases a light sedative may be administered. However, in our centre, sedation has never been required for a breast MR examination because of the favourable patient positioning. If one examines MRI of all body parts, the sedation rate is approximately $4 \%$.

Gadolinium contrast must be given intravenously during the examination. Previous allergy to Gd - DTPA is a contraindication; however, true anaphylactic shock following gadolinium administration occurs in only $0.0003 \%$ of patients (Niendorf et al, 1998).

Previous surgery, especially cardiac, may cause image distortion because of metal artefact from valves or sternal closure wire.

Absolute contraindications to MRI are mainly because of the presence of ferromagnetic materials. Any non-MRI compliant implant needs to be investigated for MRI compatibility. Any suspicion of a metal injury including foreign bodies in the eye or injuries from shrapnel needs to be excluded.

Following the initial financial outlay for an MR scanner, the running costs are still significant with an average scan costing around $£ 350$ including contrast agents.

\section{SPECIFICITY ISSUES}

MRI of the premenopausal breast may suffer reduced specificity because of the presence of 'hormone reactive' parts of the breast. These 'pseudo-lesions' enhance following Gd-DTPA variably throughout the menstrual cycle. Kuhl et al (1997) reported that the least troublesome time for scanning is within week 2 of the menstrual cycle. The use of hormone replacement therapy will also alter enhancement of the breast (Reichenbach et al, 1999).

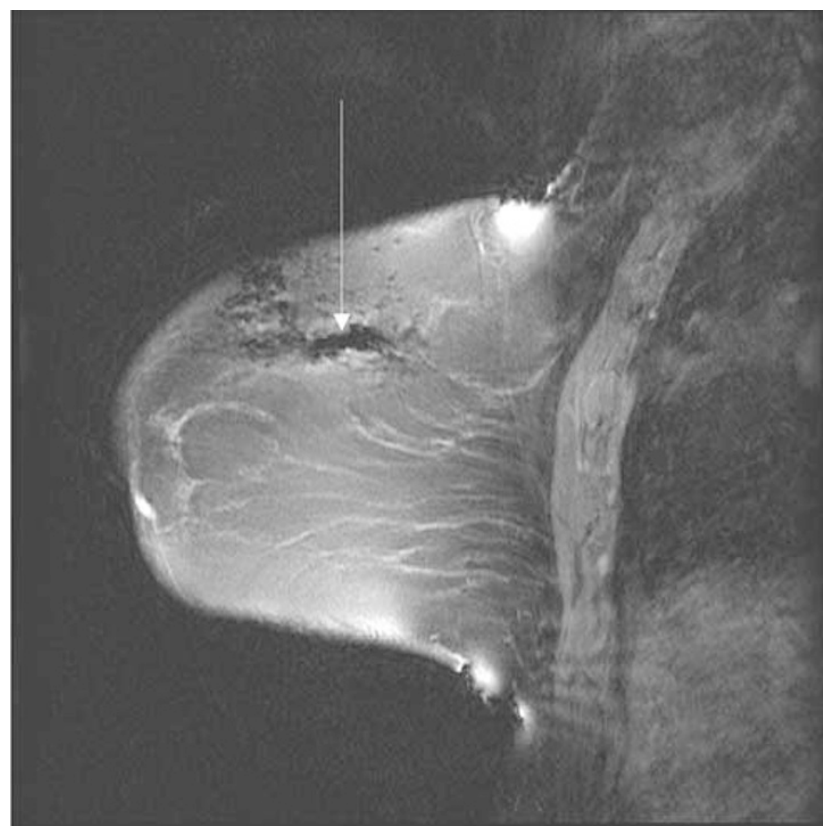

Figure 7 Tl-weighted postcontrast fat-suppressed image of a breast showing signal voids from surgical instrument metal fragments. 
Vascular fibroadenomas and papillomas form a large proportion of false positives as they can enhance in a similar way to malignant lesions. The commercially available Gd-chelates are extracellular, nontissue-specific compounds that leak out of the intravascular space. New macromolecular contrast agents leak through cancer microvessels, but do not leak through the endothelial barrier of benign tumours (Gerlowski and Jain, 1986). These blood-pool contrast agents are currently being developed. These may provide a marked improvement in the ability to examine the macrocirculation as well as microcirculation and thereby increase specificity.

Although DCE-MRI has been shown to be effective in distinguishing scar from recurrent tumour following breast surgery, it can be limited when used immediately following surgery. Interpretation of images may be compromised because of haemorrhage.

The presence of metal clips or even tiny amounts of metal rubbed off surgical instruments can severely reduce the image quality caused by metal artefact (Figure 7).

\section{FUTURE}

The progression of MR technology will uncover smaller lesions that are occult on X-ray mammography, ultrasound and clinical evaluation. 3T systems with multichannel receivers will undoubtedly increase both spatial and temporal resolutions and the use of $4 \mathrm{D}$ sequences will provide full breast coverage. Using special pulse sequences and a thin slice thickness, small lesions will be evaluated using both contrast uptake and morphologic parameters. The development of tumour-specific contrast agents may provide a simple answer to the issues of specificity. If the nature of these lesions cannot be clarified using noninvasive MR methods, then

\section{REFERENCES}

Beahrs OH, Henson DE, Hunter RVP, Kennedy BJ (1992) AJCC manual for staging of cancer. 4th edn. Philadelphia: Lippicott, 1992

Belli P, Costantini M, Romani M, Marano P, Pastore G (2002) Magnetic resonance imaging in breast cancer recurrence. Breast Cancer Res Treat 73: $223-235$

Bradley AJ, Carrington BM, Hammond CL, Swindell R, Magee B (2000) accuracy of axillary $\mathrm{MR}$ imaging in treated breast cancer for distinguishing between recurrent tumour and treatment effects: does intravenous Gd-DTPA enhancement help in cases of diagnostic dilemma. Clin Radiol 55: $921-928$

Brown J, Buckley D, Coulthard A, Dixon AK, Dixon JM (2000) Magnetic resonance imaging screening in women at genetic risk of breast cancer: imaging and analysis protocol for the UK multi-centre study. Magn Reson Imag 18: 765 - 776

Buckley DL, Drew PJ, Mussurakis S, Monson JRT, Horsman A (1997) Microvessel density in invasive breast cancer assessed by dynamic Gd DTPA enhanced MRI. J Magn Reson Imag 7: $461-464$

Buckley DL, Kerslake RW, Blackband SJ, Horsman A (1994) Quantitative analysis of multi-slice GD - DTPA enhanced dynamic MR images using an automated simplex minimization procedure. Magn Reson Imag 32: $646-651$

Drew PJ, Chatterjee S, Turnbull LW, Read J, Carleton PJ, Fox JN, Monson JRT, Kerin MJ (1999a) Dynamic contrast enhanced magnetic resonance Imaging of the breast is superior to triple assessment for the preoperative detection of multifocal breast cancer. Ann Surg Oncol 6: 599 603

Drew PJ, Kerin MJ, Mahapatra TK (2001) Evaluation of response to neoadjuvant chemoradiotherapy for locally advanced breast cancer with dynamic contrast-enhanced MRI of the breast. Eur J Surg Oncol 27: 617 620 (Abstract)

Drew PJ, Kerin MJ, Turnbull LW, Imrie M, Carleton PJ, Fox JN, Monson JRT (1998) Routine screening for local recurrence following breastconserving therapy for cancer with dynamic contrast-enhanced magnetic resonance imaging of the breast. Ann Surg Oncol 5: 265-270

Drew PJ, Turnbull LW, Chatterjee S, Read J, Carleton PJ, Fox JN, Monson JRT, Kerin MJ (1999b) Prospective comparison of standard triple the development of MR-guided biopsy or MR-guided surgery will have to continue. However, further trials are needed as to the clinical relevance of these very small invasive or in situ carcinomas especially when these are multifocal.

MRI of the breast has shown great potential in the evaluation of breast disease in research centres; however, its acceptance into common practice is limited. This may be because of the availability of MR systems with long waiting times in many centres. This may improve with the introduction of additional new opportunities funding (NOF) systems. There is also a national shortage of radiologists, especially those who specialise in MR and breast disease. Advances in software will enable compression of the huge MR data sets, particularly dynamic contrast-enhanced data with functional images corresponding to contrast uptake characteristics. This may help to reduce the time taken to report MR breast examinations.

The variety of scanning techniques and hardware limit reproducible results from centre to centre. To gain widespread usage, we must develop systems that exceed current standards of imaging but will not require complex data analysis. We must standardise protocols compatible with the hardware that is available to the majority of hospitals. In the meantime, breast MRI will continue to be regarded as a problem-solving tool.

\section{ACKNOWLEDGEMENT}

We acknowledge the support of The University of Hull Yorkshire Cancer Research. assessment and dynamic magnetic resonance imaging of the breast for the evaluation of symptomatic breast lesions. Ann Surg 230: $680-685$

Esserman L, Hylton N, Yassa L (1999) Utility of magnetic resonance imaging in the management of breast cancer: evidence for improved preoperative staging. J Clin Oncol 17: 110-119

Fischer U, Kopka L, Grabbe E (1999) Breast carcinoma: effect of preoperative contrast-enhanced MR imaging on the therapeutic approach. Radiology 213: $881-888$

Gerlowski LE, Jain RK (1986) Microvascular permeability of normal and neoplastic tissues. Microvasc Res 31: $288-305$

Gilles R, Zafrani B, Guinebretiere J, Meunier M, Lucidarme O, Tardivon A, Rochard F, Vanel D, Neuenschwander S, Arriagada R (1995) Ductal carcinoma in-situ. MR-imaging and histopathologic correllation. Radiology 196: 415 - 419

Gilles R, Meunier N, Lucudarme O (1996) Clustered breast microcalcifications: evaluation by dynamic contrast-enhanced subtraction MRI. J Comput Assist Tomogr 20: $14-15$

Gorczyca DP, DeBruhl ND, Mund DF (1994) Linguine sign at MR imaging: does it represent the collapsed silicone implant shell? Radiology 191: $576-577$

Gould SWT, Lamb G, Lomax D, Gedroyc W, Darzi A (1998) Interventional MR-guided excisional biopsy of breast lesions. J Magn Reson Imag 8: $26-30$

Hayes C, Padhani AR, Leach MO (2002) Assessing changes in tumour vascular function using dynamic contrast-enhanced magnetic resonance imaging. NMR Biomed 15: $154-163$

Heywang-Kobrunner SH, Huynh AY, Viehweg P, Hanke W, Requardt H, Paprosch I (1994) Generic prototype breast coil for MR-guided needle localisation. J Comput Assist Tomogr 18: 876-881

Heywang-Kobrunner SH, Wolf A, Pruss E, Hilbertz T, Eiermann W, Permanetter W (1989) MR imaging of the breast with Gd-DTPA: use and limitations Radiology 171: 95 - 103

Jacquillat C, Weil M, Auclerc G (1989) Neoadjuvant chemotherapy in the conservative management of breast cancer: a study of 252 patients Recent results. Cancer Res 115: 36 - 37 
Kneeshaw PJ, Gibbs P, Liney G, Lowry M, Turnbull LW, Drew PJ (2001a) Dynamic contrast enhanced MRI of sub-1 cm clinically occult Breast Lesions. Eur J Surg Oncol 27: 804 - 805 (Abstract)

Kneeshaw PJ, Lowry M, Turnbull LW, Drew PJ (2001b) Does dynamic contrast enhanced MRI have a role in the investigation of indeterminate microcalcification in the breast. Eur J Surg Oncol 27: 792 (Abstract)

Kramer S, Schulz-Wendrland R, Hagedorn K, Baitz W, Lang N (1998) Magnetic resonance imaging in the diagnosis of local recurrences in breast cancer. Anticancer Res 18: $2159-2161$

Kuhl CK, Bieling HB, Gieseke J, Kreft BP, Sommer T, Lutterby G, Schild HH (1997) Healthy pre-menopausal breast parenchyma in dynamic contrastenhanced MR imaging of the breast: normal contrast medium enhancement and cyclical-phase dependency. Radiology 203: 137 - 144

Kuhl CK, Mielcarek P, Klaschik S (1999) Dynamic breast MR imaging: are the signal intensity time course data useful for differential diagnosis of enhancing lesions. Radiology 211: $101-110$

Kuhl CK, Morakkabati N, Leutner CC, Schmiedel A, Wardelmann E, Schild $\mathrm{HH}$ (2001) MR imaging-guided large-core (14-gauge) needle biopsy of small lesions visible at breast MR alone. Radiology 220: 31 - 39 (Generic)

Liney G, Tozer DJ, Brunsveld van Hulten H, Beerens EGJ, Gibbs P, Turnbul LW (2000) Bilateral open breast coil and compatible intervention device. J Magn Reson Imag 12: 984 - 990

Liu PF, Debatin JF, Caduff RF, Kacl G, Garzoli E, Krestin GP (1998) Improved diagnostic accuracy in dynamic contrast enhanced MRI of the breast by combined quantitative and qualitative analysis. $\mathrm{Br} \mathrm{J}$ Radiol 71: $501-509$

Malich A, Boehm T, Facius M, Freesmeyer MG, Fleck M, Anderson R, Kaiser WA (2001) Differentiation of mammographically suspicious lesions: evaluation of breast ultrasound, MRI mammography and electrical impedance scanning as adjunctive technologies in breast cancer detection. Clin Radiol 56: $278-283$

Niendorf HP, Alhassan A, Balzer T, Claub W, Geens V (1998) Safety and Risk of Gadolinium-DTPA: Extended Clinical Experience After More than 20 Million Applications, 3rd edn. Berlin: Blackwell
Orel SG, Dougherty CS, Reynolds CR, Czerniecki BJ, Siegelman ES, Schnall MD (2000a) MR imaging in patients with nipple discharge: initial experience. Radiology 216: 248 - 254

Orel SG, Schnall MD, Powell CM (1995) Staging of suspected breast cancer: effect of MR imaging and MR guided biopsy. Radiology 196: $115-122$

Orel SG, Weinstein SP, Schnall MD, Reynolds C, Schuchter LM, Fraker DI (2000b) Breast MR imaging in patients with axillary node metastasis and unknown primary malignancy. Radiology 212: 543 - 549

Rankin SC (2000) MRI of the breast. Br J Radiol 73: $806-818$

Reichenbach JR, Przetac C, Klinger G, Kaiser WA (1999) Assessment of breast tissue changes on hormonal replacement therapy using MRI: a pilot study. J Comput Assist Tomogr 23: $407-413$

Roberts MM, Alexander FE, Anderson TJ (1990) Edinburgh trial of screening for breast cancer: mortality at seven years. Lancet 1: $241-246$

Stoutjesdijk MJ, Boetes C, Jager GJ, Beex L, Bult P, Hendriks JH, Laheij RJ, Massuger L, Van Die LE, Wobbes T, Barentsz JO (2001) Magnetic resonance imaging and mammography in women with a hereditary risk of breast cancer. J Natl Cancer Inst 93: 1095-1102

Tan JE, Orel SG, Schnall MD, Schultz DJ, Solin LJ (1999) Role of magnetic resonance imaging and magnetic resonance imaging-guided surgery in the evaluation of patients with early stage breast cancer for breast conservation treatment. Am J Clin Oncol 22: 414-418

Turnbull LW (2000) Magnetic Resonance Imaging: its role in the breast. Breast Cancer: Diagnosis and Management. In Dixon JM (ed). p 1-14. Amsterdam: Elsevier Science.

Vaupel PW (1994) Blood Flow, Oxygenation, Tissue pH Distribution and Bioenergetic Status of Tumours. Hellmich: Berlin

Warner E, Plewes DB, Shumak RS, Catzavelos GC, Di Prospero LS, Yaffe MJ, Goel V, Ramsay E, Chart PL, Cole DEC, Taylor GA, Cutrara M, Samuels TH, Murphy JP, Murphy JM, Narod SA (2002) Comparison of breast magnetic resonance imaging, mammography, and ultrasound for surveillance of women at high risk for hereditary breast cancer. J Clin Oncol 19: $3524-3531$

Westerhof JP, Fischer U, Moritz JD, Oestmann JW (1998) MR imaging of mammographically detected clustered microcalcifications: is there any value. Radiology 207: $675-681$ 